\title{
Erratum
}

\section{Erratum: Acharya et al., New Concerns for Neurocognitive Function during Deep Space Exposures to Chronic, Low Dose-Rate, Neutron Radiation}

In the article "New Concerns for Neurocognitive Function During Deep Space Exposures to Chronic, Low Dose-Rate, Neutron Radiation," by Munjal M. Acharya, Janet E. Baulch, Peter M. Klein, Al Anoud D. Baddour, Lauren A. Apodaca, Eniko A. Kramár, Leila Alikhani, Camillo Garcia Jr., Maria C. Angulo, Raja S. Batra, Christine M. Fallgren, Thomas B. Borak, Craig E. L. Stark, Marcello A. Wood, Richard A. Britten, Ivan Soltesz, and Charles L. Limoli, which was published online on August 5, 2019, a formula appeared incorrectly due to a production error. Within the formula on page 4, ("x 00") should be corrected to ("x 100 ").

https://doi.org/10.1523/ENEURO.0367-19.2019 